short-circuiting and opening the appendix are only makeshifts, for neither removes the cause of the intestinal stasis.

It is too early to speak definitely as to the results of these short-circuiting operations. Occasionally the operation itself is fatal. I, like some others, have seen some women who are not in the least better for having had it done; on the other hand, many successes have been reported, but sometimes more than one operation has been required, and sometimes aperients are still necessary after the operation. It would be a good thing if some of these cases were more fully reported; photographs before and a year after the operation would be useful, and the patients who had been operated on, whether suecessfully or not, might be shown before this society. It is desirable to have the after-history of every case that has ever undergone operation, for it may be that any improvement that ensues is not permanent; this is especially important, as many of the subjects of operation are neurotic women, and we all know that the immediate effects of operation on them may be extraordinary. Further, it is unfortunate that the most frequent symptom-namely, pain-is subjective.

In this brief survey $I$ have tried to indicate the direction which future work should take. I hope I have not been too critical ; if any think so I would beg them to remember that friendly criticism can do no harm. Our interest in alimentary toxæmia has been awakened by the fascinating suggestions of Metchnikoff and by the bold extension of them by Mr. Arbuthnot Lane, but it is an approved principle of science that the results of one worker cannot be accepted until confirmed by others. We ought, therefore, to withhold our verdict on many points until we see whether confirmation is forthcoming. In the year 600 A.D. Isidorus Hispalensis, Bishop of Seville, wrote: "For a medical man should know the ars rhetorica, that he may be able to support with sound arguments the matters which he deals with; and also the ars dialectica, so that by the exercise of reason he may investigate the causes of sickness for the purposes of cure." That is true now as then.

Wimpole-street, $\mathrm{W}$.

\section{THE ETIOLOGY OF CYSTS CONNECTED WITH HERNIA SACS.}

BY R. W. MURRAY, F.R.C.S. ENG., SURGEON, YORTHERT HOSPITAL, IIVERPOOL.

ANY surgeon who has had occasion to perform a large number of operations for the radical cure of inguinal hernia must, from time to time, have met with anomalous conditions of the sac and its connexions which are difficult to explain. This has been my experience, and I will exemplify my meaning by referring to two cases, and at the same time venture to offer an explanation of the state of affairs found at the operation.

In Case 1 the patient was a young man suffering with a "hydrocele of the cord." At the operation a small hernia sac was found, and connected with the fundus of the sac

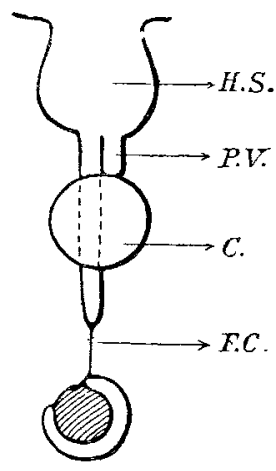

Case 1

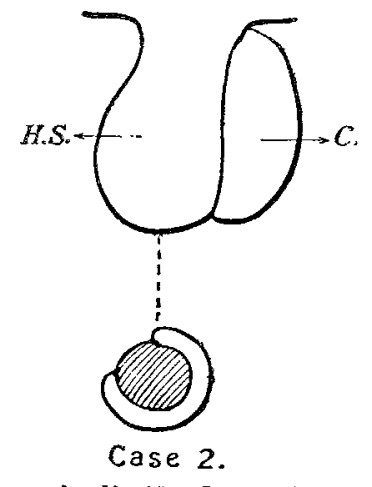

Case 2 H.S., Hernia sac. P.V., Processus vaginalis (duplicated). c., Cyst.
F.C., Fibrous cord.

was the peculiar and complicated condition depicted in the accompanying diagram. Attached to the fundus of the hernia sac were two patent funicular processes, each of which opened into the sac. At the blind end of the shorter process was a tense cyst, one inch in length, which contained clear fluid. The longer process had a uniform lumen and passed downwards behind the cyst, to which it was intimately adherent. The lower end of this process was connected with the tunica vaginalis by a fibrous cord, so that it undoubtedly was the remains of the true processus vaginalis testis.

Case 2 was that of an adult male, who was admitted to hospital for the cure of a scrotal hernia which had existed for many years and could not be reduced satisfactorily. At the operation a large cyst, which contained clear fluid, was found lying upon and intimately adherent to the anterior surface of the sac wall. The relative size and relation of the cyst to the hernia sac are illustrated in the diagram.

Though the origin of the cysts in these two cases may, at first sight, appear to have nothing in common and be difi. cult to account for, their presence can, I believe, be readily explained on the views advanced many years ago by $\mathrm{Mr}$. C. B. Lockwood regarding the etiology of infantile hernia. Mr. Lockwood pointed out that if the processus raginalis is drawn down by the gubernaculum, a second sac of peritoneum might be produced by the traction of some additional fibres at the gubernaculum in a manner entirely analogons to that which produced the processus vaoinalis testis.

It is a well-known fact that the obliteration of the processus vaginalis is frequently imperfect, and often takes place in an irregular manner. The most common defect is for the upper part of this process to remain patent, thus forming a potential hernia sac. The whole of this process may remain pervious, or the upper and lower ends become obliterated, resulting in a cystic distension of the unobliterated part. Many other irregularities have been observed, but I merely wish to emphasise the fact that obliteration may occur at any point in the whole length of this process, from the tunica vaginalis to the internal abdominal ring. In Figs. $I$ to 6 I have represented diagrammatically how the tunica vaginalis is formed, together with some of the more common developmental defects.

Bearing these facts in mind, let us see how the anomalous condition found in the two cases I have mentioned can be accounted for. The chief difficulty in the first case is to account for the presence of two funicular processes attached to the fundus of a hernial sac. It is generally acknowledged that the gubernaculum is in some way responsible for the normal descent of a process of peritoneum to the bottom of the scrotum in order to form the tunica vaginalis. Whether this portion of the peritoneum is pulled down by or merely guided by the gubernaculum I do not know, though one would expect a muscular structure to pull rather than guide. As the gubernaculum is responsible for the formation of one funicular process, it is reasonable to suppose that if this process is duplicated the gubernaculum must have something to do with it. I know of no other structure which could perform a similar function.

In the case we are now considering (Case 1) I would suggest that the peritoneal attachment of the gubernaculum was bifurcated, and as a consequence two peritoneal processes instead of a single one had been pulled through the inguinal canal towards the scrotum. (See Figs. 7 and 8.) It should be noticed that the duplication of the funicular pro. cess is not complete; that is to say, the division does not extend up to the level of the internal abdominal ring. Both processes open into a larger diverticulum. (Fig. 9.) As the duplication is not complete it is probable that the peritoneal attachments of the gubernaculum were not widely separated, and its contraction caused the funicular process of peritoneum to have two nipple-like terminations. The subsequent changes are not so difficult to account for, and are depicted in Figs. 9 to 11. The longer process, after forming the tunica vaginalis, became obliterated at its lower part into a fibrous cord, the upper part remaining pervions. The shorter process became obliterated about its middle, thus forming a closed serous sac below, and remained per. vious above. (Fig. 10.) The bowel descended into the large single diverticulum into which both these processes opened, thus converting it into a hernia sac, and fluid accumulating in the closed serous sac a cyst was formed. (Fig. 11.)

In my other case (Case 2) I believe the derelopmental irregularities were very similar to those $\mathbf{I}$ have just described. In this case, however, I would draw attention to the fact that the duplication of the processus vaginalis was more complete, extending up to the level of the internal abdominal ring. This I would account for by supposing that the upper 


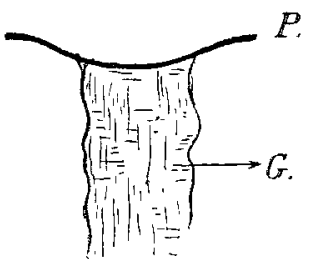

1.

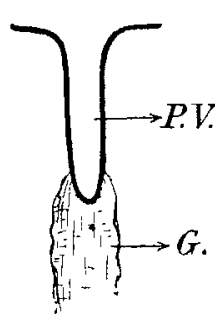

2.

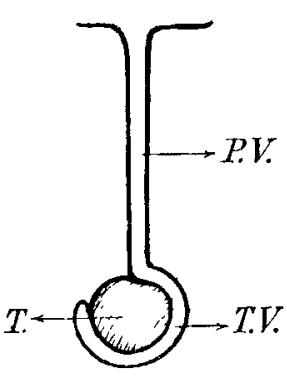

3.

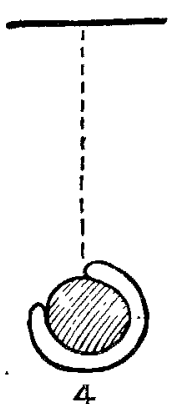

4

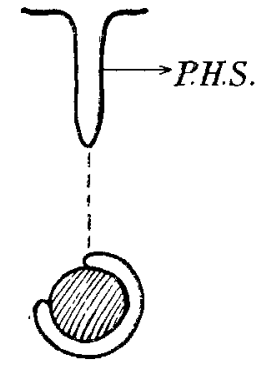

5.

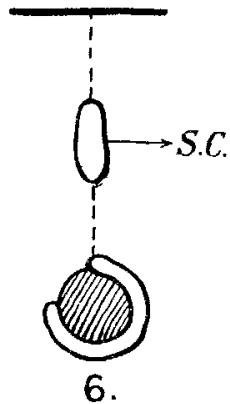

6 .

Figs. 1 to 4 represent the normal development of the tunica raginalis. In Figs. 5 and 6 a portion of the processus vaginalis testis remains umobliterated, thus forming a potential hernia sac in one case and a "hydrocele of the cord" in the other. G., Gubernaculum. P., Peritoneum. P.V.. Processus vaginalis. T., T'estis. T.V., Tunica vaginalis. P.H.S., Potential hernia sac. s.C., Serous cyst of cord.

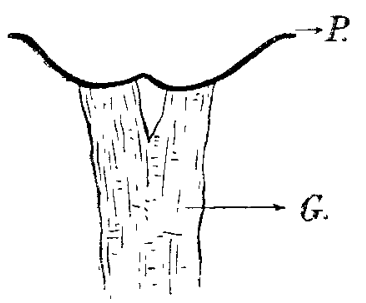

7.

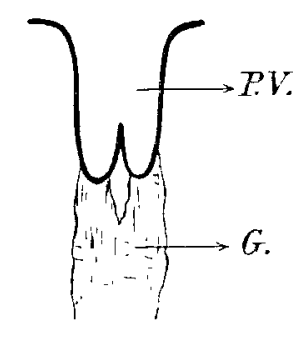

8.

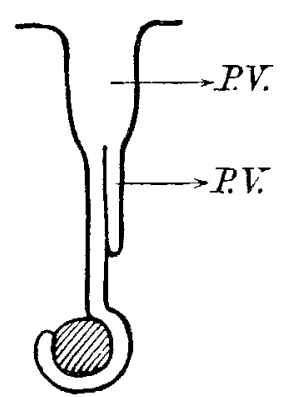

9.

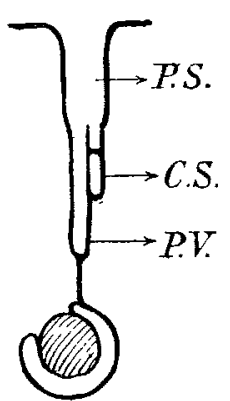

10.

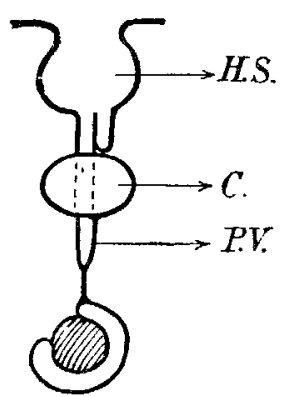

II.

Double attachment of the gubernaculum to the peritoneum, and consequent partial duplication of the processus vaginalis. P.S., Potential bernia sac. c.s., Closed sac. H.s., Hernia sac. c., Cyst.

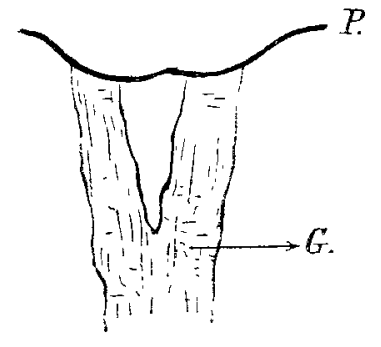

12.

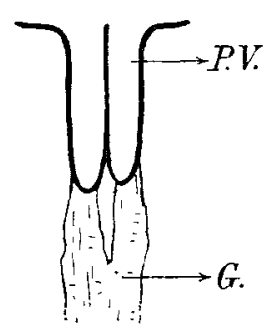

13.

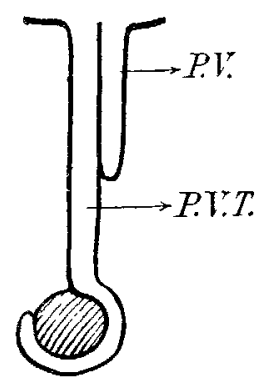

14.!

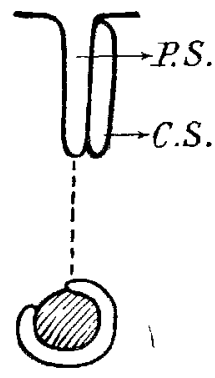

15.

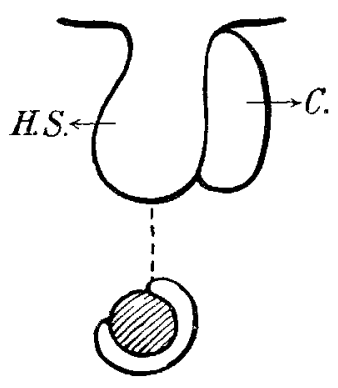

16.

Wide double attachment of the gubernaculum to the peritoneum and consequent complete duplication of the processus vaginalis.

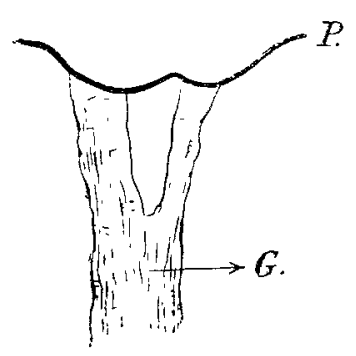

17.

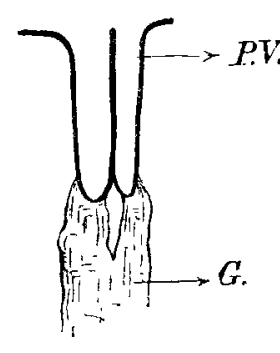

18

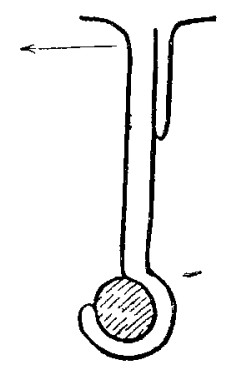

19.

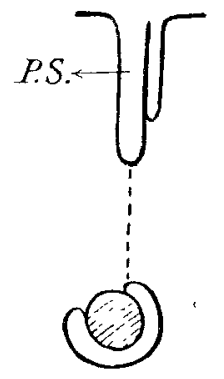

20.

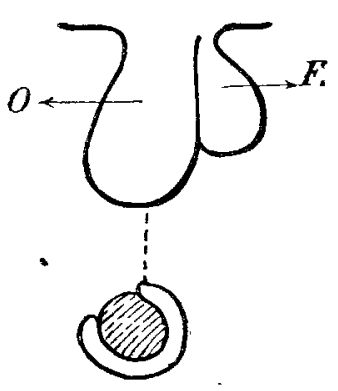

21.

Complete but unequal duplication of the processus vaginalis owing to the unequal division oflthe gubernaculum. The imperfect obliteration of these processes results in the formation of two sacs (Case 3). o., Contained omentum. F., Contained a little fluid. 
end of the gubernaculum was bifurcated, but the peritoneal attachments were more widely apart than in the other case ; consequently two complete and separate processes of peritoneum were pulled through the inguinal canal into the scrotum. (Figs. 12 and 13.) The lower half of the longer process (the true processus vaginalis testis) became obliterated after forming the tunica vaginalis, the upper half remaining pervious. The upper part of the shorter process became obliterated, thus forming a closed serous sac. (Figs. 14 and 15.) Bowel entering the patent diverticulum converted it into a hernia sac, and fluid accumulating in the closed serous sac made it a cyst. All these changes are represented diagrammatically in the accompanying figures.

Two years ago, during an operation for the cure of an inguinal hernia in a young man $I$ found two sacs intimately attached one to the other. The larger sac contained adherent omentum, the other, which was mnch smaller, contained only a little fluid. (Case 3, Figs. 17 to 21.) In this case an explanation similar to that I have advanced would also account for this developmental irregularity.

In conclasion, I would submit that as the gubernaculum is undoubtedly responsible for the presence of the peritoneal diverticulum known as the processus vaginalis testis, circumstantial evidence is strongly in favour of the view that if this process is found to be duplicated then this second diverticulum must be due to a duplication of the force which produced the first. If this premise be correct then the subsquent irregular obliteration of these processes clearly accounts for the anomalous conditions I have described.

Liverpool.

\section{SPASTIC CYCLOPHORIA.}

\section{BY A. ALISON BRADBURNE, F.R.C.S. EDIN.}

THE effects which it is possible for uncorrected small degrees of astigmatism to produce have within recent years become fully realised by the general body of practitioners, but little account is taken of the effect produced by astigmatism when it happens to be obliquely situated.

Astigmatism when either at $90^{\circ}$ or $180^{\circ}$ produces blurring of vertical or horizontal lines which can be corrected by ciliary action or may even be passed over and compensated for by mental correction. When, however, it happens to lie obliquely there becomes superimposed on the blurring the distortion or obliquity of the originally horizontal or vertical line. Thus a line of words on a page in a book becomes sloping or a rectangular figure appears oblong and its angles obtuse or acute. Before such an object can be viewed with both eyes correctly it is necessary for the eyes to rotate around a fore-ancl-aft axis to form a correct single mental impression. As the need for such action on the part of the eyes arises from a mental requirement and the centre governing its correction belongs to the higher and more specialised ones, it is obvious that the effects which can be produced by oblique astigmatism are greater, more exacting, and profounder than those produced by rectangularly placed errors.

It is not uncommon to meet with cases of long-standing neurasthenia, of unassignable cause, completely cured by simple correction of a small degree of oblique astigmatism. It is advisable in all cases of nervous debility to exclude the possibility of such a condition, even with the history of having had the eyes tested, as not only may it have been overlooked, but an oblique astigmatism may have supervened owing to a change in the refraction, or it may even have been added owing to incorrect glassing. The account of the following case and its critical examination proves these points in a way which but few afford, and it is hoped that it will assist the elucidation of similar and more obscure cases which at present are far too commonly neglected, if not aggravated through ignorance concerning the subject.

The patient, aged 32, unmarried, was sent to me by her medical attendant in October, 1910 . Her general complaint was one of poor ness of nerve tone, together with occasional headaches and ocular strain, but not of any marked intensity. She had worn glasses constantly for over a year, which, though at first affording relief, had never been particularly comfortable. It is important to note what these glasses were. In the right eye there was a plus $0.5 \mathrm{D}$. sph. combined with a myopic cylinder of $1 \mathrm{D}$. axis $30^{\circ}$ down and in. In front of the left eje was a plus $0.25 \mathrm{D}$. sph. combined with a plus cylinder at axis $30 \circ$ down and out-that is to say, the glasses made the right eye one of mixed oblique, and the left eye one of hypermetropic oblique astigmatism. Now refraction and testing proved both eyes. were in reality the subject of oblique hypermetropic astigmatism, of amount of $0.5 \mathrm{D}$. at axis $45^{\circ}$ down and in in each eye, both axis and amount being equal in each eye. With the cylinders correcting this astigmatism-that is, at axis $45^{\circ}$ out-in the trial frame and a Maddox double prism before one eye, the test for cyclophoria was carried out by asking the patient to view and state the position of the three lines produced by the prism and the unglassed eye. The result of the test was that the centre line was seen to be sloping down and out towards the temple. The cylinders were then slowly rotated, and it was seen that when they were moved nearer the vertical--that is, towards $90^{\circ}$-the sloping of the central line became greater, whilst when they were rotated in the opposite direction-that is, towards $180^{\circ}$-the sloping lessened. The patient was ordered fius $0.5 \mathrm{D}$. cylinders at axis $45^{\circ}$ down and out in each eye with the most gratifying result.

From the above account arise three questions which call for explanatory answers. 1. The interpretation of the sloping line presented by the double prism? 2. The reason for the alteration produced on the central line by moving the cylinders? 3 . The cause for the anomalous position of the eyes?

1. In regard to the first question, the meaning of the sloping of the central line. Here, although the line appeared to slope, it could not but be associated with a

FIG. 1.

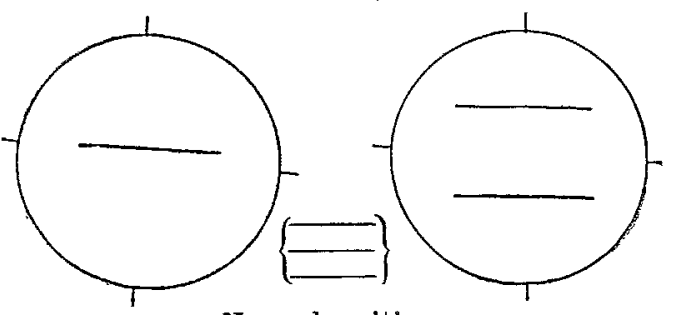

Normal position.

FIG. 1A.
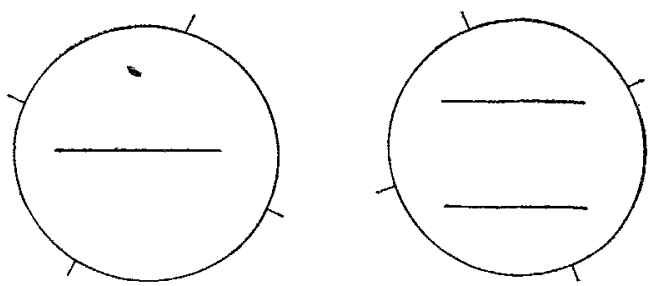

Vertical meridians intorted. Actual position.

FIG. 1B.

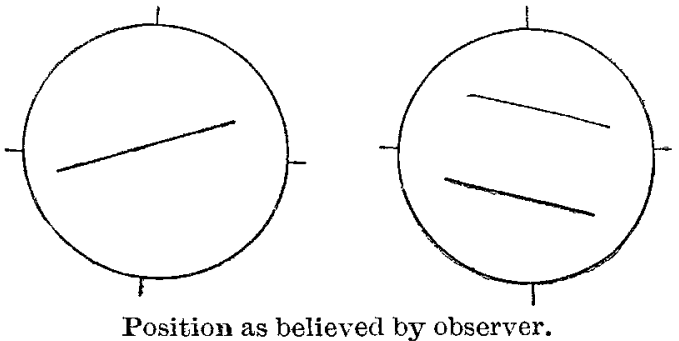

defect in the position of the eye, as the line itself was truly horizontal. This line, it was stated by the patient, sloped down and out towards the temple. Remembering the fact that the line is really horizontal, such a slope could only be imagined by the line falling on an eye which was. rotated inwards towards the nose, and its false interpretation arises because the patient imagines her eye to be horizontal, and therefore ascribes the tilt to the line and not to her eye. The true position of the eyes, then, was one of intortion.

2. It may now be inquired why movement of the cylinders affected the tilt of the line. Turning for a moment to the refraction of the eyes, we find that they are the subject of oblique hypermetropic astigmatism, and, as already pointed out, this condition produces a displacement of the retinal images, such as that of a horizontally placed picture of an arrow. The rule which obtains in cases of oblique hypermetropic astigmatism is that the displacement of the retinal image takes place in a directic n towards the meridian of greatest curvature, which in the case under discussion is seen to be at $45^{\circ}$ down and in. The effect of such retinal displacement would be to give an impression of crossed or scissor-like appearance which the fusion faculty would not tolerate, and would make a call on the centre 